\title{
Light-induced electron localization in a quantum Hall system
}

\author{
T. Arikawa ${ }^{1 \star}$, K. Hyodo', Y. Kadoya ${ }^{2,3}$ and K. Tanaka $a^{1,3,4}$
}

An insulating bulk state is a prerequisite for the protection of topological edge states ${ }^{1}$. In quantum Hall systems, the thermal excitation of delocalized electrons is the main route to breaking bulk insulation ${ }^{2}$. In equilibrium, the only way to achieve a clear bulk gap is to use a high-quality crystal under high magnetic field at low temperature. However, bulk conduction could also be suppressed in a system driven out of equilibrium such that localized states in the Landau levels are selectively occupied. Here we report a transient suppression of bulk conduction induced by terahertz wave excitation between the Landau levels in a GaAs quantum Hall system. Strikingly, the Hall resistivity almost reaches the quantized value at a temperature where the exact quantization is normally disrupted by thermal fluctuations. The electron localization is realized by the long-range potential fluctuations, which are a unique and inherent feature of quantum Hall systems. Our results demonstrate a new means of effecting dynamical control of topology by manipulating bulk conduction using light.

The integer quantum Hall effect (IQHE) is a universal phenomenon observable in two-dimensional electron systems under high magnetic field $(B)$. The Hall resistivity $\left(\rho_{x y}\right)$ is quantized and the longitudinal resistivity $\left(\rho_{x x}\right)$ becomes zero irrespective of the detailed properties of the host materials ${ }^{2-7}$. This universality has lead to a new physical concept of topological invariant that defines the quantized value of $\rho_{x y}$ (ref. 8). In systems with sample boundaries, edge metallic states appear according to the bulk/edge correspondence ${ }^{9}$ and carry quantized Hall current. The edge states are protected by the bulk gap between the Landau levels (LLs), which prohibits the scattering between macroscopically separated edge states $^{10,11}$. In realistic situations, we need to take into account the impurity and finite-temperature effect. The LLs are broadened with delocalized states in the centre, sandwiched by localized states induced by Anderson localization ${ }^{12,13}$ (Fig. 1d). At finite temperatures, the delocalized states are broadened and thermally populated, which makes the bulk conductive $e^{2,14}$. As a result, the scattering between edge states is allowed and $\rho_{x y}\left(\rho_{x x}\right)$ is no longer quantized (zero).

In this paper, we demonstrate a transient suppression of bulk conduction and a recovery of the quantized $\rho_{x y}$ by selectively populating the localized electronic states. A direct way to manipulate the electron distribution in LLs is inter-LL transition by resonant light absorption (typically in the terahertz frequency region). To verify this strategy for realizing light-induced IQHE, we performed transient Hall measurements on a quantum Hall system with a non-equilibrium electron distribution induced by picosecond terahertz pulse excitation. Previous research of this kind ${ }^{15-21}$ was done in an ideal quantum Hall state and explained by the heating effect due to the nanosecond to microsecond terahertz pulses they employed. Therefore, the idea of light-induced IQHE has not been explored yet.

Before studying the terahertz excitation effect, the basic characterization of the sample was performed at the equilibrium state. The solid blue curves in Fig. 1a show $\rho_{x x}$ (left) and $\rho_{x y}$ (right) as a function of $B$ measured at $4.3 \mathrm{~K}$ with a bias current $\left(i_{\mathrm{S}}\right)$ of $10 \mu \mathrm{A}$. The partial plateaux in $\rho_{x y}$ and the corresponding minima in $\rho_{x x}$ are developed around even filling factors $(v)$. The non-zero $\rho_{x x}$ even at $v=6$ is due to the finite-temperature effect: $\rho_{x x}$ increases as the number of delocalized electrons $\left(N_{\text {deloc }}\right)$ increases. At $v=6, \rho_{x x}$ is simply proportional to $N_{\text {deloc }}$. This relationship is evident in the thermal activation behaviour of $\rho_{x x}(T) \propto N_{\text {deloc }}(T) \propto \exp \left(-E_{\mathrm{A}} / k_{\mathrm{B}} T\right)$, where $E_{\mathrm{A}}$ is the activation energy and $k_{\mathrm{B}}$ is the Boltzmann constant ${ }^{14}$ (Fig. 1b,d). The larger $i_{\mathrm{S}}$ heats up the electron temperature $\left(T_{\mathrm{e}}\right)$. The dashed blue curve in Fig. 1a shows $\rho_{x x}$ at $4.3 \mathrm{~K}$ measured with $i_{\mathrm{S}}=100 \mu \mathrm{A}$. The $\rho_{x x}$ value at $\nu=6$ is much larger and $T_{\mathrm{e}}$ determined from the activation law is $12.8 \mathrm{~K}$ (blue circle in Fig. 1b). Similar experiments are performed with different bias currents to obtain the bias current dependence of the electron temperature, which is shown in Fig. 1c.

Next we show the results of the terahertz excitation effect. As shown above, $\rho_{x x}$ is a direct probe of the electron localization state. Figure 2a shows the transient differential longitudinal resistivity $\left(\Delta \rho_{x x} / \rho_{x x}\right)$ after terahertz pulse (inset in Fig. 2a) excitation at $4.3 \mathrm{~K}$. The experiments were done at $4.0 \mathrm{~T}(\nu=6.5)$, where the bulk is conducting and $\rho_{x x}\left(\rho_{x y}\right)$ largely deviates from zero $\left(h / 6 \mathrm{e}^{2}\right)$. Also, $\rho_{x x}$ at $4.3 \mathrm{~K}$ and $8.3 \mathrm{~K}$ intersects at $4.0 \mathrm{~T}$ (Fig. 1a). This enables us to eliminate the heating effect and study the non-equilibrium electron distribution effect. The blue curve was measured with $i_{\mathrm{S}}=$ $54 \mu \mathrm{A}$. The longitudinal resistivity increases by $30 \%$ immediately after the resonant inter-LL excitation, which means the electrons are delocalized and the bulk becomes more conducting. The excited state, however, does not simply decay. Instead, $\Delta \rho_{x x} / \rho_{x x}$ becomes negative (down to $-30 \%$ ) after $0.3 \mu$ s, suggesting a significant amount of electrons are localized and the bulk conduction is suppressed. The localized state lasts for a few microseconds.

The terahertz-induced electron delocalization and the subsequent localization can be understood by considering carrier dynamics in a random potential, a unique feature to a quantum Hall system. First, strong terahertz excitation induces Landau ladder climbing ${ }^{22}$ (Fig. 3a,b and Methods), which is followed by rapid (within the time resolution of $\sim 25 \mathrm{~ns}$ ) intra-LL thermalization (Fig. 3c). Also, ultrafast superradiant decay ${ }^{23}$ (estimated decay time $\sim 10 \mathrm{ps}$ ) should also happen. Note that possible coherent effects such as Floquet-Bloch

\footnotetext{
${ }^{1}$ Kyoto University, Department of Physics, Kyoto 606-8502, Japan. ${ }^{2}$ Hiroshima University, Graduate School of Advanced Science of Matter, Higashihiroshima 739-8530, Japan. ${ }^{3}$ CREST, Japan Science and Technology Agency, Tokyo 102-1075, Japan. ${ }^{4}$ Kyoto University, Institute for Integrated Cell-Material Sciences (WPI-iCeMS), Kyoto 606-8501, Japan. *e-mail: arikawa@scphys.kyoto-u.ac.jp
} 

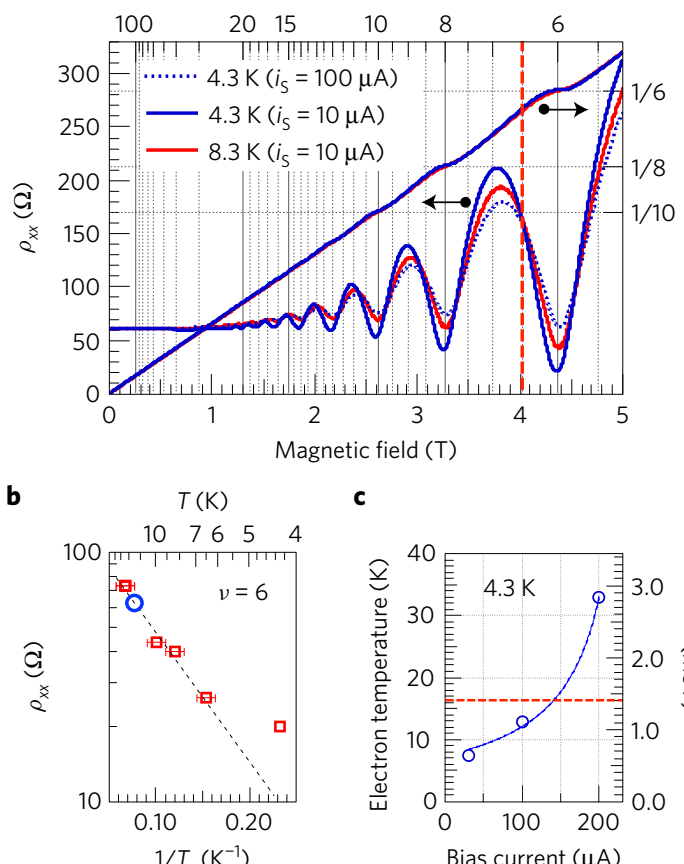

Filling factor

c

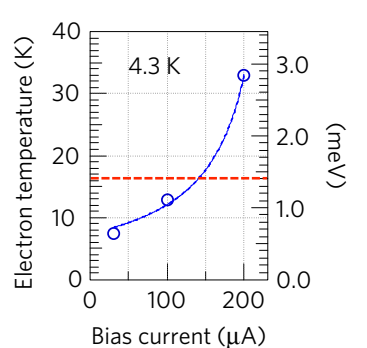

d

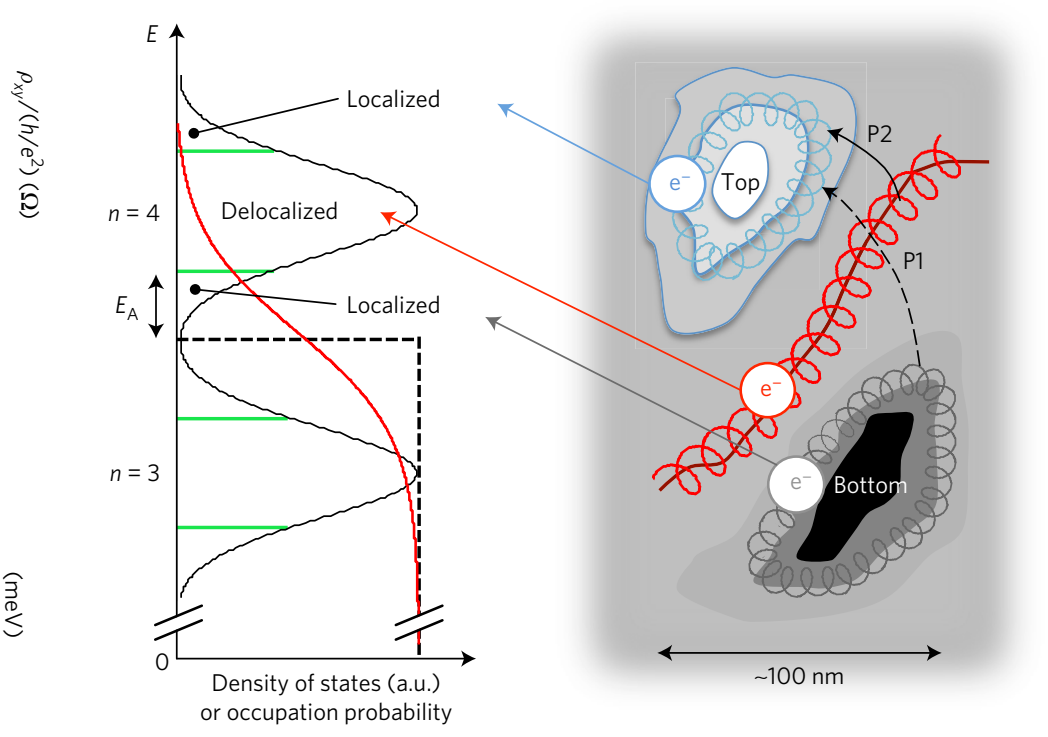

Figure 1 | Quantum Hall effect at thermal equilibrium. a, Longitudinal ( $\rho_{x x}$ left) and Hall ( $\rho_{x y}$, right) resistivity measured at three different conditions (lattice temperature and bias current). The corresponding filling factor $\left(\nu=h n_{\mathrm{e}} / \mathrm{e} B\right.$ ) is shown as the top horizontal axis, where $h$ is the Planck constant, $n_{\mathrm{e}}$ is the electron density, and $e$ is the elementary charge. The vertical broken line indicates a fixed point of $\rho_{x x}$ at 4.0 T. b, Temperature dependence of $\rho_{x x}$ at $\nu=6$ measured with $i_{S}=10 \mu \mathrm{A}$ showing the thermal activation behaviour (straight broken line) with $E_{\mathrm{A}}=1 \mathrm{meV}$. The deviation at $4.3 \mathrm{~K}$ is due to the variable range hopping ${ }^{14}$. The blue circle shows $\rho_{x x}$ measured at $4.3 \mathrm{~K}$ with $i_{s}=100 \mu \mathrm{A}$ indicating the electron temperature of $12.8 \mathrm{~K}$. The error bars represent the temperature stability of $\pm 0.5 \mathrm{~K}$. c, Bias current dependence of the electron temperature. The lattice temperature is $4.3 \mathrm{~K}$. The right axis shows the electron temperature in meV unit. The broken curve (blue) is a theoretical fitting (see Methods). The dashed line (red) represents the half-width at half-maximum of the LLs $(1.4 \mathrm{meV})$. d, Schematic picture of the density of states (left) and electron drift motion in real space with a long-range potential fluctuation (right). The localized electrons (blue and grey $\mathrm{e}^{-}$) perform drift motion along closed equipotential lines around a potential minimum and maximum separated by $\sim 100 \mathrm{~nm}$ (refs 2,13). The delocalized states (red $\mathrm{e}^{-}$) only exist between them where equipotential lines are extended over the finite-sized sample. These localized and delocalized states in real space can be mapped to the energy position within LLs as shown by three straight arrows. The mobility edges (green lines) separate the localized and delocalized states. The activation energy $E_{\mathrm{A}}$ defines the position of the mobility edge relative to the chemical potential. The dashed black (solid red) curve shows the Fermi-Dirac distribution function at $0 \mathrm{~K}$ (finite-temperature) when $v=6$, showing the thermal excitation of the delocalized states. $n$ is the spin-degenerated LL index. P1 and P2 show two relaxation pathways described in the text.

a

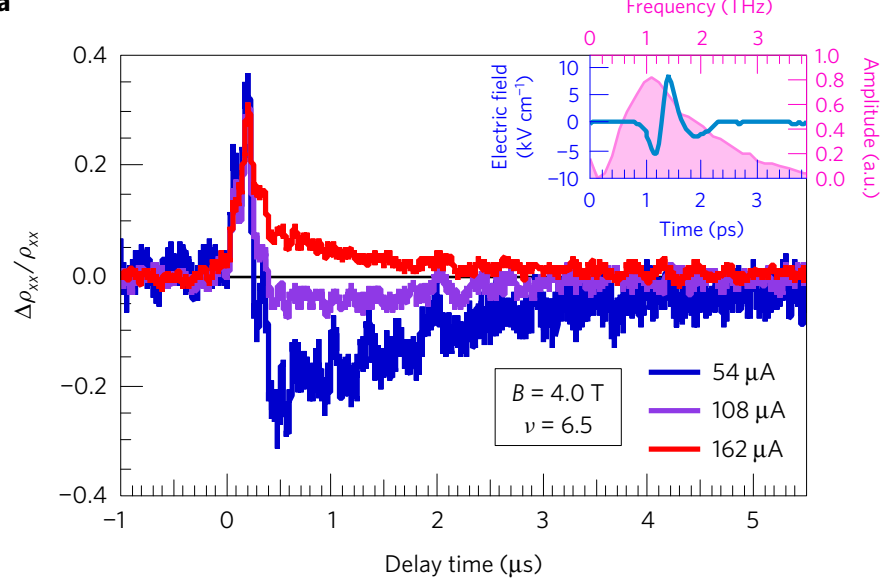

b

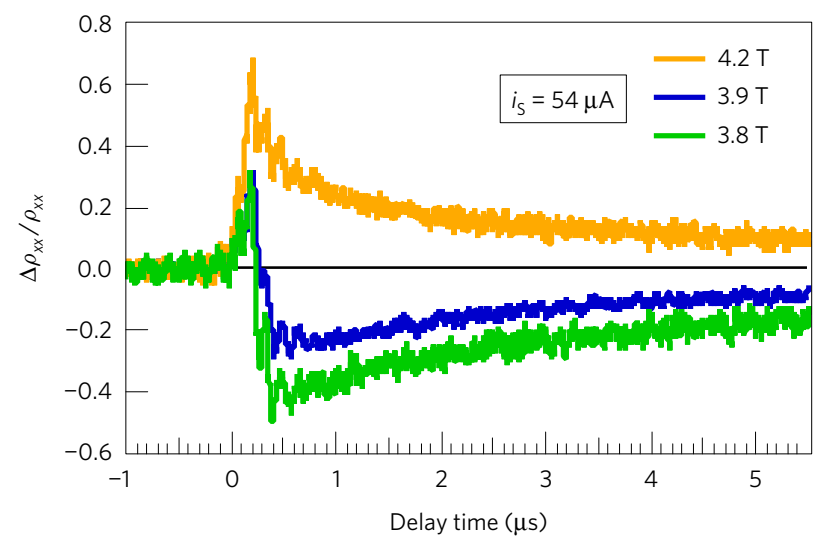

Figure 2 | Terahertz-pump DC-probe experiments at $4.3 \mathrm{~K}$. a, Transient differential longitudinal resistivity at 4.0 T $(v=6.5)$ measured with different bias currents (see Methods and Supplementary Information 3 for data treatment). These signals show different decay dynamics although the initial increase due to the inter-LL transition is almost constant (30\%). The inset shows the waveform (light blue) and spectrum (magenta) of the terahertz pulse that excites the sample at delay time zero. b. Transient differential longitudinal resistivity at different magnetic fields around $4.0 \mathrm{~T}$ measured with a fixed bias current of $54 \mu \mathrm{A}$. The longer decay than $5 \mu$ s reflects the lattice cooling time. The initial increase of $30 \%$ is also observed at 3.8 and $3.9 \mathrm{~T}$, whereas at $4.2 \mathrm{~T}$ the lattice heating effect significantly increases the overall signal. 
a

$E(\mathrm{meV})$

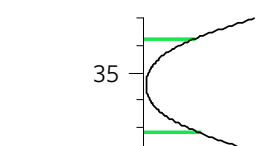

$(n=5)$

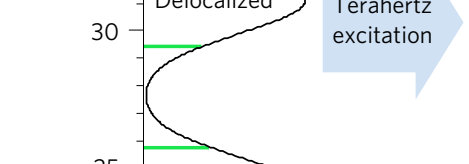

$(n=4)$

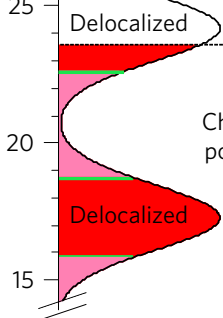

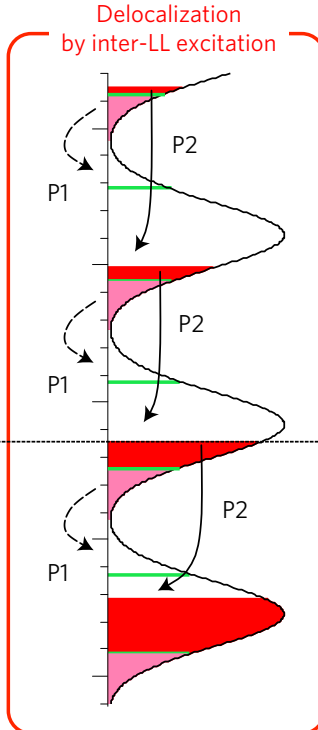

d

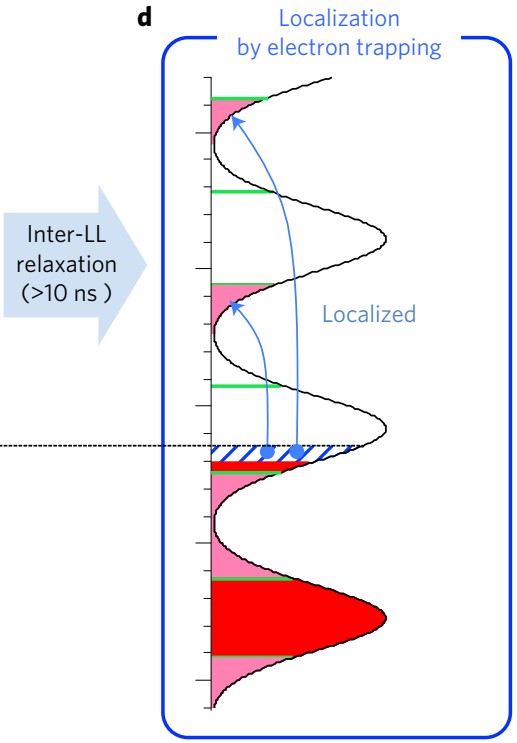

Figure 3 | Electron localization mechanism after terahertz excitation. a, Electron distribution over LLs at 4.0 T ( $\nu=6.5)$ before the terahertz excitation. The shape of the density of states is approximated by a Gaussian function with a half-width at half-maximum of 1.4 meV, which is estimated from the Dingle analysis ${ }^{29}$ (see Supplementary Information 2). The area in magenta, defined by the mobility edges (green lines), represents localized electrons. For the sake of clarity, the green lines are drawn closer to the centre of each $L L$ than reality. We assume $T_{\mathrm{e}}=0 \mathrm{~K}$ for simplicity. $\mathbf{b}$, Inter-LL transition to the higher levels induced by intense terahertz wave excitation ${ }^{22}$. Since the momentum of the terahertz photon is negligible, inter-LL excitation happens between states with the same centre-of-mass position. This is represented by the black vertical arrows (with the length equal to the cyclotron energy) that connect states with the same spatial position (see also Fig. 1d). c, Electron distribution after rapid (femtosecond to picosecond) intra-LL thermalization. We assumed the same electron temperature defined in a (before the terahertz excitation), which can be controlled by the bias current is. Even the localized electrons in the upper tail of each LL are thermalized before slow (nanosecond) inter-LL relaxation starts. Two pathways (inefficient P1 and efficient P2) for inter-LL relaxation are shown. $\mathbf{d}$, Transient electron localized state. The electrons in $n=5,6, \ldots$ LLs are trapped around the potential minimum.

state formation ${ }^{24,25}$ would be undetectable due to the poor time resolution. In Fig. 3c, delocalized electrons at quasi-Fermi levels not only in $n=4$, but also in other LLs contribute bulk conduction, which explains the positive $\Delta \rho_{x x} / \rho_{x x}$. Afterwards, the excited electrons should undergo inter-LL relaxation to the equilibrium state by emitting acoustic phonons in 10 to $100 \mathrm{~ns}$ (refs 26,27). However, a simple relaxation never explains the electron localization. We propose the following electron localization mechanism. The important point is the existence of the highly forbidden relaxation pathway, P1: relaxation of localized electrons in the lower tail of a LL to localized states in the upper tail of the LL below (Fig. 3c). This is because the centre positions of electrons in the initial and final states are spatially separated by $\sim 100 \mathrm{~nm}$ (ref. 13), which is larger than the spatial extent of the wavefunction (magnetic length) $\sim 10 \mathrm{~nm}$ (Fig. 1d). Thus, according to the standard microscopic theory of electronphonon interaction, the transition probability between these states is vanishingly small $\left(\sim \exp \left[-(100 / 10)^{2} / 4\right]\right)$ due to little spatial overlap of the wavefunction ${ }^{28}$. The localized electrons in the lower tail have to relax to the lower tail of the LL below, but Pauli blocking forbids this process, which results in their long lifetime. In contrast, the relaxation path $\mathrm{P} 2$ : relaxation between delocalized states near the quasi-Fermi levels should be more efficient because these states are spatially closer. As a consequence, the electrons near the quasiFermi levels relax quickly and a non-equilibrium distribution like Fig. $3 \mathrm{~d}$ should be realized during the relaxation. Here, localized states in $n=5,6, \ldots$ LLs are selectively populated by electrons that were originally in the $n=4$ delocalized states (blue hatched region in Fig. 3d). The observed relaxation time of the localized state in Fig. 2a (a few microseconds) is comparable to the previously reported value on a similar trapped state ${ }^{20}$, which supports the above localization mechanism.

We experimentally examined this mechanism in the following way. The key process is the preferential inter-LL relaxation from the upper delocalized states. This would not be prominent when the thermal broadening $\left(k_{\mathrm{B}} T_{\mathrm{e}}\right)$ of the electron distribution is comparable to, or larger than the half-width at half-maximum of the LLs $(1.4 \mathrm{meV})$. Here, $T_{\mathrm{e}}$ is the effective temperature defined by the quasi-equilibrium electron distribution after intra-LL thermalization (Fig. 3c), which should be almost the same as the original electron temperature defined in Fig. 3a. In this case, the electrons at the bottom of the potential can be thermally excited inside each LL and relax via path P2. As a result, electrons relax randomly independent of their positions within LLs and a normal decay from the delocalized state (Fig. 3c) to the original state (Fig. 3a) is expected. To verify this, we performed similar experiments while raising $T_{\mathrm{e}}$ by increasing $i_{\mathrm{S}}$ (purple and red curves in Fig. 2a). As clearly seen, electron localization is suppressed with $108 \mu \mathrm{A}\left(k_{\mathrm{B}} T_{\mathrm{e}} \sim 1.1 \mathrm{meV}\right.$, see Fig. 1c) and completely gone with $162 \mu \mathrm{A}\left(k_{\mathrm{B}} T_{\mathrm{e}} \sim 1.7 \mathrm{meV}\right)$. On the other hand, the positive peak value is independent of $i_{s}$. This also indicates the initial delocalization is due to the inter-LL transition by the constantpower terahertz excitation.

The acoustic phonon emission during the inter-LL relaxation heats up the lattice and electron temperature. This effect on $\rho_{x x}$ starts to dominate when $B$ deviates from $4.0 \mathrm{~T}$. Figure $2 \mathrm{~b}$ shows transient $\Delta \rho_{x x} / \rho_{x x}$ at 3.8, 3.9 and 4.2 T $\left(\nu=6.9,6.7\right.$ and 6.2) with $i_{\mathrm{S}}=54 \mu \mathrm{A}$. In all cases, the same processes depicted in Fig. 3 (with slightly different chemical potentials) should happen. As expected, similar dynamics are observed at 3.8 and 3.9 T, but with much slower decay than at $4.0 \mathrm{~T}$. According to Fig. 1a, electron heating lowers $\rho_{x x}$ at 3.9 $\mathrm{T}$ and most drastically at $3.8 \mathrm{~T}$. Thus, the negative signal consists of both the electron localization and heating effect, and the slow decay is attributed to the lattice cooling time. In contrast, at $4.2 \mathrm{~T}$, $\rho_{x x}$ increases by electron heating (Fig. 1a), which would compensate the negative signal due to electron localization. As shown in Fig. 2b, $\Delta \rho_{x x} / \rho_{x x}$ at $4.2 \mathrm{~T}$ does not show a negative signal, which means the 


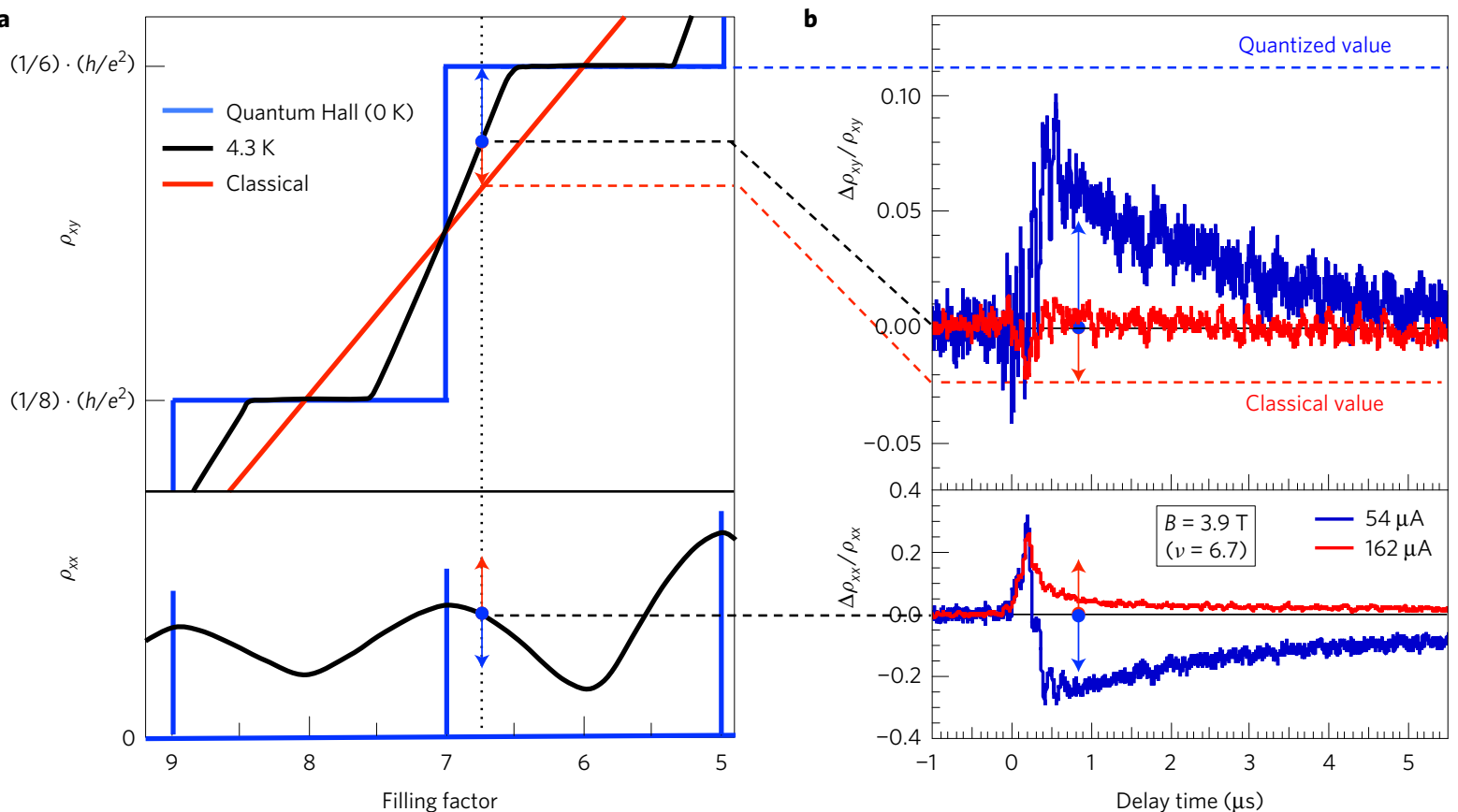

Figure 4 | Transient recovery of the topological edge states. a, Schematic picture of the Hall (upper) and longitudinal (lower) resistivities as a function of the filling factor $v$. The spin splitting is neglected. The plateau width gets narrower as the delocalized states becomes wider at high temperatures. b. Simultaneous measurement of the transient Hall and longitudinal resistivities at 3.9 T $(v=6.7)$. The blue (red) dashed line shows the quantized (classical) value of the Hall resistivity. The blue arrows represent the recovery of the topological edge states due to the electron localization. The red arrows represent electron delocalization that makes system classical.

heating effect is dominant. The slow decay also indicates the positive signal is due to the lattice heating effect.

Finally, we show how the transient suppression of the bulk conduction modifies the Hall resistivity (blue curves in Fig. 4b). The transient $\Delta \rho_{x y} / \rho_{x y}$ shows a positive signal while electron localization is observed in $\Delta \rho_{x x} / \rho_{x x}$. This means that $\rho_{x y}$ is approaching the quantized value, as shown by the blue arrow in Fig. 4a. Here, blue and red lines schematically show the ideal quantum Hall and classical system, respectively, while the black line between them represents our data taken at $4.3 \mathrm{~K}$. Strikingly, $\rho_{x y}$ almost reaches the quantized value $\left(h / 6 \mathrm{e}^{2}\right.$, blue dashed line). This clearly demonstrates that the system is transiently recovering the topological edge states by non-equilibrium selective population of the localized states. With $i_{\mathrm{S}}=162 \mu \mathrm{A}$ (red curves in Fig. $4 \mathrm{~b}$ ), where electron localization is not observed in $\rho_{x x}$, we only see an initial decrease in $\rho_{x y}$ that reaches the classical value (red dashed line). This suggests that the system is almost classical because electrons are delocalized. Note that the initial decrease in $\rho_{x y}$ is also observed in the blue curve because of the common delocalization process (Fig. 3b,c).

\section{Methods}

Methods, including statements of data availability and any associated accession codes and references, are available in the online version of this paper.

Received 22 December 2015; accepted 23 February 2017; published online 3 April 2017

\section{References}

1. Ando, Y. Topological insulator materials. J. Phys. Soc. Jpn 82, 102001 (2013).

2. Yoshioka, D. The Quantum Hall Effect (Springer, 2010).

3. Klitzing, K. v., Dorda, G. \& Pepper, M. New method for high-accuracy determination of the fine-structure constant based on quantized Hall resistance. Phys. Rev. Lett. 45, 494-497 (1980).

4. Wakabayashi, J. \& Kawaji, S. Hall effect in silicon MOS inversion layers under strong magnetic fields. J. Phys. Soc. Jpn 44, 1839-1849 (1978).
5. Paalanen, M. A., Tsui, D. C. \& Gossard, A. C. Quantized Hall effect at low temperatures. Phys. Rev. B 25, 5566(R)-5569(R) (1982).

6. Novoselov, K. S. et al. Two-dimensional gas of massless Dirac fermions in graphene. Nature 438, 197-200 (2005).

7. Xu, Y. et al. Observation of topological surface state quantum Hall effect in an intrinsic three-dimensional topological insulator. Nat. Phys. 10, 956-963 (2014).

8. Thouless, D. J., Kohmoto, M., Nightingale, P. \& den Nijs, M. Quantized Hall conductance in a two-dimensional periodic potential. Phys. Rev. Lett. 49, 405-408 (1982).

9. Hatsugai, Y. Chern number and edge states in the integer quantum Hall effect. Phys. Rev. Lett. 71, 3697-3700 (1993).

10. Aoki, H. Localisation in the quantum Hall regime. Surf. Sci. 196, 107-119 (1988).

11. Ibach, H. \& Lüth, H. Solid-State Physics: An Introduction to Principles of Materials Science (Springer, 2009).

12. Ando, T. Electron localization in a two-dimensional system in strong magnetic fields. II. long-range scatterers and response functions. J. Phys. Soc. Jpn 53, 3101-3111 (1984).

13. Hashimoto, K. Quantum Hall transition in real space: from localized to extended states. Phys. Rev. Lett. 101, 256802 (2008).

14. Wei, H. P., Chang, A. M., Tsui, D. C. \& Razeghi, M. Temperature dependence of the quantized Hall effect. Phys. Rev. B 32, 7016(R)-7019(R) (1985).

15. Maan, J. C., Englert, Th., Tsui, D. C. \& Gossard, A. C. Observation of cyclotron resonance in the photoconductivity of two-dimensional electrons. Appl. Phys. Lett. 40, 609-610 (1982).

16. Lavine, C. F., Wagner, R. J. \& Tsui, D. C. Magnetic field dependence of the photoresponse of the electron inversion layer on (100) Si. Surf. Sci. 113, 112-117 (1982).

17. Stein, D., Ebert, G., Klitzing, K. v. \& Weimann, G. Photoconductivity on GaAs- $\mathrm{Al}_{x} \mathrm{Ga}_{1-x}$ As heterostructures. Surf. Sci. 142, 406-411 (1984).

18. Rikken, G. L. J. A. et al. Nanosecond far infra red magnetospectroscopy of GaAs/AlGaAs heterostructures. Surf. Sci. 196, 303-309 (1988).

19. Dießel, E. et al. Novel far-infrared-photoconductor based on photon-induced interedge channel scattering. Appl. Phys. Lett. 58, 2231-2233 (1991).

20. Kawano, Y., Hisanaga, Y., Takenouchi, H. \& Komiyama, S. Highly sensitive and tunable detection of far-infrared radiation by quantum Hall devices. J. Appl. Phys. 89, 4037-4048 (2001).

21. Kalugin, N. G. et al. Different components of far-infrared photoresponse of quantum Hall detectors. Appl. Phys. Lett. 81, 382-384 (2002). 
22. Maag, T. et al. Coherent cyclotron motion beyond Kohn's theorem. Nat. Phys. 12, 119-123 (2016).

23. Zhang, Q. et al. Superradiant decay of cyclotron resonance of two-dimensional electron gases. Phys. Rev. Lett. 113, 047601 (2014).

24. Wang, Y. H., Steinberg, H., Jarillo-Herrero, P. \& Gedik, N. Observation of Floquet-Bloch states on the surface of a topological insulator. Science 342, 453-457 (2013)

25. Fregoso, B. M., Wang, Y. H., Gedik, N. \& Galitski, V. Driven electronic states at the surface of a topological insulator. Phys. Rev. B 88, 155129 (2013).

26. Smirnov, K. et al. Energy relaxation of two-dimensional electrons in the quantum Hall effect regime. JETP Lett. 71, 31-34 (2000).

27. Hirakawa, K. \& Sakaki, H. Energy relaxation of two-dimensional electrons and the deformation potential constant in selectively doped AlGaAs/GaAs heterojunctions. Appl. Phys. Lett. 49, 889-891 (1986).

28. Dietzel, F., Dietsche, W. \& Ploog, K. Electron-phonon interaction in the quantum Hall effect regime. Phys. Rev. B 48, 4713-4720 (1993).

29. Coleridge, P. T. Small-angle scattering in two-dimensional electron gases. Phys. Rev. B 44, 3793-3801 (1991).

\section{Acknowledgements}

This work was supported by the KAKENHI (26247052) from Japan Society for the Promotion of Science (JSPS)

\section{Author contributions}

T.A. and K.H. performed the measurements and analysed the data. Y.K. prepared the sample studied. T.A. and K.T. prepared the manuscript. All authors discussed the results and contributed to this manuscript.

\section{Additional information}

Supplementary information is available in the online version of the paper. Reprints and permissions information is available online at www.nature.com/reprints. Publisher's note: Springer Nature remains neutral with regard to jurisdictional claims in published maps and institutional affiliations. Correspondence and requests for materials should be addressed to T.A.

\section{Competing financial interests}

The authors declare no competing financial interests. 


\section{Methods}

Sample. The sample was a two-dimensional electron gas in a GaAs/AlGaAs single heterostructure. We used a Hall bar geometry $(W=200 \mu \mathrm{m}$ width and

$L=1,200 \mu \mathrm{m}$ length, see Supplementary Information 1) to measure Hall and longitudinal resistivity using a four-terminal method with a sourcemeter (input resistance $>10 \mathrm{G} \Omega$ ) or an oscilloscope ( $50 \Omega$ input resistance). The electron density and mobility at $4.3 \mathrm{~K}$ were $6.2 \times 10^{11} \mathrm{~cm}^{-2}$ and $1.7 \times 10^{5} \mathrm{~cm}^{2} \mathrm{~V}^{-1} \mathrm{~s}^{-1}$, respectively. The mobility was almost constant between $4.3 \mathrm{~K}$ and $15 \mathrm{~K}$.

Bias current dependence of the electron temperature. We evaluated the bias current dependence of the electron temperature (broken blue curve in Fig. 1c) as follows. Below the breakdown current threshold, $\rho_{x x}$ exponentially increases with the bias current; $\rho_{x x} \propto \exp \left[-\left(A_{1}-A_{2} i_{\mathrm{S}}\right) / k_{\mathrm{B}} T\right]$, where $A_{1}$ and $A_{2}$ are constants $^{30}$. By equating this expression to $\exp \left(-E_{\mathrm{A}} / k_{\mathrm{B}} T_{\mathrm{e}}\right)$, we obtain $T_{\mathrm{e}}=\left(A_{3}-A_{4} i_{\mathrm{S}}\right)^{-1}$, where $A_{3}$ and $A_{4}$ are the fitting parameters in Fig. 1c.

Terahertz-pump DC-probe measurement. The sample was mounted in an optical cryostat with a superconducting magnet to apply a perpendicular magnetic field to the two-dimensional electron gas. The Faraday geometry was used. Picosecond coherent terahertz pulses were generated from gas plasma created by focusing the fundamental $(800 \mathrm{~nm})$ and second harmonic $(400 \mathrm{~nm})$ pulses of a $100 \mathrm{fs}$ Ti:S regeneratively amplified laser. The terahertz pulses are almost linearly polarized along the Hall bar. A silicon wafer with a thickness of $2 \mathrm{~mm}$ was used to block the residual 800 and $400 \mathrm{~nm}$ beams propagating collinearly with the generated terahertz pulses to avoid optical breakdown of the quantum Hall state. The cryostat window was covered with another silicon wafer to put the sample in a dark condition and avoid photo-carrier generation. See Supplementary Information 1 for the experimental schematics. The frequency spectrum covers the cyclotron resonance frequencies in the experiment ( 1.6 to $1.7 \mathrm{THz}$ or 6.6 to $7.0 \mathrm{meV}$ ) to induce inter-LL transition. The intensity of the terahertz pulse is kept constant in all the experiments. The diameter of the terahertz pulses on the sample surface was $\sim 1.5 \mathrm{~mm}$ and excites almost all parts of the sample. The transient resistivity measurement was performed using a digital oscilloscope (50 $\Omega$ input resistance) while constant bias voltage was supplied (see Supplementary Information 3 for data analysis). The bias current, $i_{\mathrm{s}}$, is determined by the current-limiting resistor $(500 \mathrm{k} \Omega)$ connected in series with the sample $(\sim 1 \mathrm{k} \Omega)$. The temporal resolution was limited by the $R C$ time of the circuit and estimated to be 25 ns (assuming 500 $\mathrm{pF}$ stray capacitance). We raised the electron temperature by increasing the bias current at a lattice temperature of $4.3 \mathrm{~K}$. This is because the lattice temperature stability between 5 and $15 \mathrm{~K}$ was not good enough to perform transient Hall measurements with a sufficient signal-to-noise ratio.

Landau ladder climbing. In Fig. 3b,c we assumed that many electrons are excited to higher LLs (Landau ladder climbing) by the terahertz pulse excitation. Although we do not have direct experimental evidence, such an assumption is made plausible by the following two reasons. First, the light-matter interaction in this system (cyclotron resonance in GaAs two-dimensional electron gas) is very strong due to the enormous dipole moment $\mu_{n, n+1}=e \sqrt{\left(\hbar /\left(2 m^{*} \omega_{c}\right)\right)(n+1)}$ that reaches as large as tens of $e$-ångström, where $n$ is the LL index. This originates from the small effective mass $m^{*}\left(\sim 0.067 m_{0}\right)$ and small resonance frequency $\omega_{\mathrm{c}}(\sim 1.6 \mathrm{THz}$ in Fig. 3). Therefore, this system has been one of the best samples for exploring ultrastrong non-perturbative light-matter coupling ${ }^{22,31}$. An order-of-magnitude estimate with the assumption of $20 \%$ linear absorption with a $0.1 \mathrm{THz}$ linewidth ${ }^{32}$ gives $0.97 \times 10^{11} \mathrm{~cm}^{-2}$ for the number of excited electrons. Here, we used the following parameters given in Fig. 2a inset and Methods; $8.7 \mathrm{kV} \mathrm{cm}^{-1}$ peak electric field $\left[E\left(t_{\text {peak }}\right)\right], 1$ ps pulse duration $(\Delta t), 1.5 \mathrm{~mm}^{2}$ spot size and $1.6 \mathrm{THz}$ photon energy. This number is comparable to the LL degeneracy at $4 \mathrm{~T}\left(0.96 \times 10^{11} \mathrm{~cm}^{-2}\right)$ and indicates that the lower LLs are significantly depopulated. In such a strong excitation condition, the interaction between the two-dimensional electron gas and terahertz radiation must be treated as a coherent interaction due to the long coherence time (a few picoseconds). In a textbook two-level system, such a strong excitation leads to Rabi oscillation. In LLs, however, we have infinite ladder of LLs, and electron excitation to higher LLs in a cascading fashion is expected.

We estimated the number of excitation steps $\Delta n$ based on the following simple classical model. We assumed electrons in LLs as a classical harmonic oscillator and treated the interaction between electrons and a terahertz pulse as an impulse at the bottom of the harmonic potential. This is a reasonable assumption because the terahertz pulse width $(\Delta t \sim 1 \mathrm{ps})$ is much shorter than the coherence time $(1 / \gamma \sim$ a few picoseconds) and therefore the electron motion would be ballistic. The equation of motion is given as follows:

$$
m^{*}\left[\ddot{x}(t)+\gamma \dot{x}(t)+\omega_{\mathrm{c}}^{2} x(t)\right]=-\sqrt{f} e E_{\mathrm{CRA}}(t)
$$

where $f$ is the oscillator strength and $E_{\mathrm{CRA}}(t)=E(t) / 2$ is the amplitude of the cyclotron resonance active mode (circular polarization). For perfect harmonic oscillators, such as LLs in parabolic semiconductors, the oscillator strength is unity for all the energy levels. In the Fourier domain, we obtain the following relation,

$$
\tilde{x}(\omega)=\frac{-e}{m^{*}} \frac{\tilde{E}_{\mathrm{CRA}}(\omega)}{-\omega^{2}-i \gamma \omega+\omega_{\mathrm{c}}^{2}}
$$

Since $\hbar \omega_{\mathrm{c}}=1.6 \mathrm{THz} \gg \hbar \gamma \sim 0.1 \mathrm{THz}, \tilde{x}(\omega)$ can be converted to a delta function as follows:

$$
\tilde{x}(\omega) \approx \frac{-e}{m^{*}} \frac{\tilde{E}_{\mathrm{CRA}}(\omega)}{2 \omega_{\mathrm{c}}} \operatorname{Im}\left[\frac{1}{\omega_{\mathrm{c}}-\omega-i \gamma / 2}\right] \approx \frac{-e}{m^{*}} \frac{\tilde{E}_{\mathrm{CRA}}(\omega)}{2 \omega_{\mathrm{c}}} i \pi \delta\left(\omega-\omega_{\mathrm{c}}\right)
$$

which gives by the inverse Fourier transformation,

$$
x(t)=\frac{1}{2 \pi} \frac{-e}{m^{*}} \frac{\tilde{E}_{\mathrm{CRA}}\left(\omega_{\mathrm{c}}\right)}{2 \omega_{\mathrm{c}}} i \pi \mathrm{e}^{i \omega_{\mathrm{c}} t}
$$

Since the expectation values of the kinetic and potential energies are the same for harmonic oscillators, the total energy gained by an electron is

$$
E^{\mathrm{tot}}=2 \times \frac{1}{2} m^{*} \omega_{\mathrm{c}}^{2} \overline{x^{2}(t)}=\frac{1}{32} \frac{\mathrm{e}^{2}}{m^{*}}\left|\tilde{E}_{\mathrm{CRA}}\left(\omega_{\mathrm{c}}\right)\right|^{2}
$$

where the overbar denotes time averaging. $\left|\tilde{E}_{\mathrm{CRA}}\left(\omega_{\mathrm{c}}\right)\right|^{2}$ can be evaluated by the peak electric field in time domain using Parseval's theorem,

$$
\left|\tilde{E}_{\mathrm{CRA}}\left(\omega_{\mathrm{c}}\right)\right|^{2}=\pi(\Delta t)^{2}\left|E_{\mathrm{CRA}}\left(t_{\text {peak }}\right)\right|^{2}
$$

Here, we assumed a Gaussian pulse shape $(\Delta v \Delta t=1 / \pi)$. We can estimate the number of excitation steps as follows; $\Delta n=E^{\text {tot }} /\left(\hbar \omega_{c}\right) \approx 7.3$. Let us compare this number to the one calculated by the full quantum theory ${ }^{22}$. In ref. 22, they achieved Landau ladder climbing by nine steps using terahertz pulses with a very similar peak electric field amplitude of $8.7 \mathrm{kV} \mathrm{cm}^{-1}$ as ours. This indicates the validity of our classical modelling. This is the first reason why we naturally expect Landau ladder climbing is induced in our case.

The second reason comes from our experimental data. The data (for example Fig. 2a) show the longitudinal resistivity increases by $\sim 30 \%$ after the terahertz pulse excitation. However, the true peak value must be much larger because of the insufficient bandwidth of the oscilloscope $(400 \mathrm{MHz})$ to resolve the ultrafast population change induced by picosecond terahertz pulses. The true initial peak should rise in a few picoseconds and partially decays in $\sim 10$ ps due to the superradiance ${ }^{23}$ before slower decay ( $\left.\sim 10 \mathrm{~ns}\right)$ by acoustic phonon emission starts. Since the superradiance is very efficient ${ }^{23}$, a large amount of the population should relax in the initial $\sim 10$ ps decay, which is not detected in our experiments. Therefore, we believe that the true peak value is at least several times or one order of magnitude larger than the experimentally observed one. Such a huge change in the longitudinal resistivity is possible only when the electrons are excited to higher LLs; delocalized electrons at quasi-Fermi levels in many LLs should contribute bulk conduction.

Quantitative calculation of the population distribution after a single terahertz pulse excitation would be possible following ref. 22. In our case, however, multiple replica terahertz pulses (separated by a few picoseconds in time) created through the internal reflection inside silicon plates and cryostat windows (Supplementary Fig. 1) coherently interact with the sample within the time resolution of the measurement system $(\sim 25 \mathrm{~ns})$. This makes the numerical calculation of the initial population distribution (Fig. 3c) extremely demanding ${ }^{22}$. Even in such a situation, many higher LLs should be populated unless the very rare case of complete population quench ${ }^{32}$ happens.

Data availability. The data that support the plots within this paper and other findings of this study are available from the corresponding author upon reasonable request.

\section{References}

30. Komiyama, S., Takamasu, T., Hiyamizu, S. \& Sasa, S. Breakdown of the quantum Hall effect due to electron heating. Solid State Commun. 54, 479-484 (1985).

31. Zhang, Q. et al. Collective non-perturbative coupling of $2 \mathrm{D}$ electrons with high-quality-factor terahertz cavity photons. Nat. Phys. 12, 1005-1011 (2016).

32. Arikawa, T. et al. Quantum control of a Landau-quantized two-dimensional electron gas in a GaAs quantum well using coherent terahertz pulses. Phys. Rev. B 84, 241307(R) (2011). 